\section{Equidade e inclusão de grupos sociais na política de saúde: o caso do Recife, Brasil}

\section{Equity and the inclusion of social groups in health policy: the case of the Brazilian city of Recife}

Maria do Socorro Veloso de Albuquerque 1

André Monteiro Costa 2

Luci Praciano Lima 3

Djalma Agripino de Melo Filho 4

\begin{abstract}
Objectives: to analyze the conception and operationalization of the principle of equity in the Municipal Health Secretary's recognition of the needs of social groups in Recife between 2001 and 2008.

Methods: a case study involving semi-structured interviews and analysis of documents was carried out. The subjects were health service managers. Four social groups were selected: working people, people with psychiatric disorders, women, and people living in poverty. Thematic content analysis was used to investigate the following categories: social participation, technical and policy rationality, and integration.

Results: the Health Secretary's health policy includes various underprivileged groups, allocating resources that are not restricted to basic care, although there is a lack of communication between the various levels of care. Limitations that especially need to be overcome include: the Family Health Program referring an excessive number of patients to specialized care, suggesting problems with the identification/incorporation of users needs; occupational health policy having failed to consolidate health surveillance; failure to make progress in developing care in the community programs for people with psychiatric disorders; difficulty in meeting the diversity of needs of women, beyond pregnancy and childbirth; patchy training of health professionals in the areas of gender, race, ethnicity and class.

Conclusions: Recife's health authorities have adopted a concept of vertical equity by including diverse social groups with diverse needs, although it has difficulty in attending these needs in an integrated fashion.
\end{abstract} Key words Equity, Health policy, Population groups
${ }_{1}^{1}$ Centro de Pesquisas Aggeu Magalhães. Fundação Oswaldo Cruz. Av. Prof. Moraes Rego, 1235. Cidade Universitária. Recife, PE, Brasil. CEP: 50.670- 000. E-mail: soveloso@oi.com.br

2 Departamento de Saúde Coletiva. Centro de Pesquisas Aggeu Magalhães. Fundação Oswaldo Cruz. Recife, PE, Brasil.

3 Departamento de Medicina Social. Universidade Federal de Pernambuco. Recife, PE, Brasil.

4 Núcleo de Saúde Pública e Desenvolvimento Social. Universidade Federal de Pernambuco. Recife, PE, Brasil.

\section{Resumo}

Objetivos: analisar a concepção e operacionalização do princípio da equidade no reconhecimento de necessidades de grupos sociais pela Secretaria Municipal de Saúde (SMS) do Recife entre 2001-2008.

Métodos: estudo de caso com triangulação de técnicas (entrevistas semiestruturadas e análise documental). Sujeitos: gestores da SMS. Selecionaram-se quatro grupos sociais: trabalhadores, portadores de sofrimento psíquico, mulheres e pobres. Utilizou-se a análise temática de conteúdo para analisar as categorias: participação social, racionalidade técnica-política e integralidade.

Resultados: a política de saúde da SMS incluiu diversos grupos sociais mais desassistidos, alocando recursos não restritos à atenção básica, porém com frágil articulação entre níveis de atenção, destacando-se limitações a superar: o Programa de Saúde da Família apresenta excesso de encaminhamentos à atenção especializada, indicando problemas na identificação/incorporação das necessidades dos usuários; a política de saúde do trabalhador não consolidou ações de vigilância em saúde; foi incipiente o processo de desospitalização/desinstitucionalização dos portadores de sofrimento psíquico; foi além das ações relativas à gravidez/parto, mas enfrenta dificuldades para atender a diversidade de necessidades das mulheres; são pontuais as capacitações para os profissionais de saúde em gênero, raça, etnia e classe.

Conclusões: a SMS do Recife adotou a concepção de equidade vertical ao incluir diversos grupos sociais com necessidades distintas, porém apresenta dificuldades em atendê-las na perspectiva de integralidade da atenção. Palavras-chave Equidade, Política de saúde, Grupos populacionais 


\section{Introdução}

Mesmo com os avanços advindos com a implantação do Sistema Único de Saúde (SUS), como a expansão de oferta e cobertura dos serviços, ainda persistem, segundo Viana et al.,1 acentuadas desigualdades em relação à distribuição dos recursos e às oportunidades de acesso e utilização desses serviços entre regiões, estados, municípios e grupos sociais. Essa constatação contribui para que a equidade seja alvo de preocupações dos gestores em saúde, tanto pela magnitude das desigualdades sociais, quanto pela escassez frequente dos recursos financeiros que impõem um processo de definição de prioridades. As necessidades de serviços de saúde ou necessidades de saúde, como ressaltam Vieira-da-Silva et al.,2 variam de pessoa a pessoa e o desafio está em não impor modelos, mas definir um padrão tecnicamente aceitável para interagir com as expectativas dos diversos modos de vida de grupos sociais.

A concepção de equidade defendida pela reforma sanitária 3,4 abrange o reconhecimento das necessidades específicas dos grupos sociais e o atendimento de suas particularidades na perspectiva de políticas universais e redistributivas, não destinadas exclusivamente a grupos mais vulneráveis. Essa perspectiva implica tratar as iniquidades como desigualdades evitáveis, injustas e desnecessárias, como enfatiza Whitehead. 5

Em termos conceituais, a equidade envolve várias dimensões e por isso suscita um rico debate em sua operacionalização.2,4 Tanto se aproxima do princípio da igualdade de acesso (equidade horizontal), quanto do princípio da diferença quando reconhece a diversidade das necessidades dos sujeitos sociais (equidade vertical). No âmbito da saúde, é considerada horizontal quando se pretende identificar e tratar igualitariamente pessoas com necessidades iguais de saúde e vertical quando trata de modo diferente pessoas com necessidades distintas de saúde. 6

Autores como Sen ${ }^{7}$ ressaltam a importância de considerar múltiplos critérios na distribuição dos recursos, incluindo aspectos vinculados a gênero, etnia, idade, pobreza e sua possível interrelação. Para esse autor, pelo caráter multidimensional do conceito, a equidade pressupõe não somente questões vinculadas à distribuição das ações de atenção à saúde, mas também vislumbra a possibilidade de conquistar boa saúde. Por outro lado, sabese que a inclusão de princípios de equidade na formulação de uma política de saúde não assegura uma implementação equânime, no sentido do atendimento da diversidade de necessidades dos sujeitos. ${ }^{8}$
De acordo com Travassos et al., ${ }^{9}$ a problemática da equidade tem sido estudada na maioria das vezes sob duas dimensões: relação das condições de vida e saúde (foco nos determinantes sociais) e acesso e utilização dos serviços de saúde. O presente estudo abordou o conceito de equidade na perspectiva de inclusão de grupos sociais com necessidades distintas que demandam atenção diferenciada.

A investigação consubstanciou-se em torno de três categorias: participação social, racionalidade técnica e política e integralidade. A primeira e a segunda expressam o processo decisório para incluir ou não grupos sociais numa política de saúde ${ }^{10,11}$ e a terceira, informa o modo como o governo satisfez as necessidades desses grupos. ${ }^{12,13}$

Concebe-se a participação social como estratégia da sociedade para alterar as relações de poder a fim de alcançar maior equidade e justiça social. 10 No setor saúde, esse princípio constitui um dos indicadores da democratização do processo decisório no âmbito das políticas públicas, operacionalizado em várias instâncias, incluindo Conselhos e Conferências de Saúde. Atualmente, alguns movimentos sociais e organizações não governamentais têm-se articulado com os dirigentes governamentais de forma direta para influenciarem ações, programas e políticas. 11

A gestão em saúde é permeada por uma racionalidade técnica e política que tem forte influência na inclusão de grupos sociais. Segundo Paim,14 na saúde, o conhecimento epidemiológico, juntamente com outras racionalidades, como a política, médicoassistencial, econômica e burocrática, influenciam o processo decisório nas instituições de saúde. No entanto, se reconhece que mesmo subsidiadas por informações técnicas, as decisões em última instância são políticas, 15 refletindo escolhas dentre interesses, às vezes conflituosos, de grupos sociais.

O conceito de integralidade é polissêmico, 12,13 apresentando vários significados, dentre os quais se ressaltam: a) integração das ações de promoção, proteção, recuperação e reabilitação, compondo níveis de atenção primária, secundária e terciária; b) forma de atuação profissional abrangendo as dimensões biológica, psicológicas e sociais; c) garantia de continuidade da atenção nos diversos níveis de complexidade do sistema, que demanda uma articulação entre serviços; d) conjunto de articulação de políticas públicas. No âmbito das políticas específicas, a integralidade em seus vários sentidos pode subsidiar o entendimento das respostas governamentais à demanda e/ou necessidades de certos grupos.

Este estudo teve como principal objetivo analisar como a Secretaria Municipal de Saúde do Recife 
(SMS) concebeu e operacionalizou o princípio da equidade no reconhecimento e no atendimento de necessidades de grupos sociais.

\section{Métodos}

A pesquisa se desenvolveu no campo da análise de política, motivando a utilização do estudo de caso. Segundo Yin, 16 esse desenho é muito utilizado para compreender a emergência a e a consolidação de uma política: seus determinantes, processo de implementação e seus resultados. Adicionalmente, essa estratégia possibilita lidar com uma ampla variedade de evidências, como documentos, entrevistas e observações. Utilizou-se a abordagem qualitativa para analisar, em profundidade, a inclusão e o atendimento de necessidades de grupos sociais inseridos na política de saúde da SMS do Recife.

A pesquisa teve como espaço de observação a política de saúde do município de Recife no período de 2001-2008, pela prioridade governamental dada às questões vinculadas ao setor saúde e pela equidade aparecer como princípio norteador da política de saúde de acordo com os planos municipais de saúde do período estudado.17,18

Na seleção dos grupos sociais sobre os quais a investigação deteve maior atenção, observaram-se alguns aspectos como o acesso da população aos serviços de saúde antes do período estudado, a reivindicação dos movimentos sociais, o próprio compromisso político da gestão em incluir determinados grupos em sua política e as definições resultantes do orçamento participativo. Quatro grupos sociais foram selecionados: as mulheres, os trabalhadores, os portadores de sofrimento psíquico e os pobres.

Embora do ponto de vista conceitual seja mais fácil enquadrar os três primeiros, o mesmo não ocorre com o último deles, uma vez que "pobres" se trata mais de uma noção do que um conceito. Neste estudo, concebeu-se como "pobres" os habitantes de áreas nas quais as necessidades básicas, incluindo acesso aos serviços de saúde, não eram satisfeitas. Essas áreas, construídas a partir de um conjunto de indicadores de condições de vida, são assinaladas no mapa do desenvolvimento humano da cidade do Recife. 19

Para compor a triangulação, foram utilizadas diferentes fontes de informação e técnicas de coletas de dados (análise documental e entrevista semiestruturada). ${ }^{16} \mathrm{Na}$ análise documental, examinaram-se: planos municipais de saúde de 2002-2005 e de 20062009; relatórios de gestão da SMS (2001-2008); relatórios de políticas específicas (trabalhador 2006-
2008; mulher 2001-2008; portadores de sofrimento psíquico 2001-2004; usuários de álcool e outras drogas 2007; negros 2001-2008); caderno do perfil epidemiológico da criança e adolescente do Recife (2001-2007); relatório da vigilância à saúde da SMS sobre a situação da variável raça (2007); dados dos sistemas de informação ambulatorial (SIA-SUS), hospitalar (SIH-SUS) e sobre nascidos vivos (Sinasc).

Para as entrevistas, selecionaram-se seis informantes-chave (coordenadores e gerentes) da Secretaria de Saúde do Recife, de acordo com os critérios: compor o núcleo gestor do nível central e distrital da SMS e inserção nas duas gestões municipais durante o período de 2001-2008. As entrevistas seguiram um roteiro previamente estabelecido, foram gravadas e transcritas. Cada entrevistado recebeu uma codificação (número arábico) para garantir o anonimato. Os dados obtidos foram analisados pela técnica de análise temática de conteúdo. Foram feitas leituras exaustivas para identificação das unidades naturais de análise sintetizadas em temas. Posteriormente, procedeu-se à condensação de significados, conforme propõe Kvale.20 No processo de análise, enfatizaram-se os determinantes da inclusão dos grupos na política de saúde e as dificuldades operacionais na satisfação das necessidades de cada grupo. Além disso, considerou-se a articulação entre as políticas, ações e serviços na perspectiva de integralidade da atenção.

A pesquisa foi aprovada pelo Comitê de Ética em Pesquisa/CEP do Centro de Pesquisa Aggeu Magalhães (parecer n 21/2009).

\section{Resultados e Discussão}

Os resultados serão apresentados em tópicos nos quais cada grupo social é analisado mediante as categorias teóricas selecionadas: participação social, racionalidade técnica e política e integralidade.

\section{O enfrentamento da desassistência nas áreas}

\section{de pobreza}

O município, que contava com 16 Unidades de Saúde da Família (USF) em 2000, ampliou o número de unidades para 112 USF em 2008,21 distribuídas nas 66 zona especial de interesse social (ZEIS) do Recife ( $80 \%$ das favelas) e em outras áreas de pobreza (Figura 1). Assim, a cobertura do Programa de Saúde da Família (PSF), que era de 6\% em 2000, elevou-se para $52 \%$ em 2008 , atingindo, portanto, uma cobertura média superior à nacional no mesmo período que era de 49,5\%.22 Nas entrevistas, 
destacou-se que as áreas de pobreza foram consideradas prioritárias pela gestão:

Era preciso promover uma inversão de prioridades e atender as áreas que historicamente viam sendo desassistidas. [...] Na saúde a gente começou a expandir o processo de entrada do sistema de saúde pelo PSF nas áreas descobertas, que eram de pobreza e aí a gente começava a trazer as outras políticas para dentro do território (Entrevistado 5).

Embora essa inclusão de grupos na assistência básica tenha ocorrido nas áreas de pobreza, os percentuais de cobertura foram diferentes entre os distritos sanitários (com variação entre 39\% e 64\% em 2008)21 e essa oscilação não obedeceu a nenhum critério de estratificação de pobreza entre os distritos. Assim, verifica-se que o uso da racionalidade técnica seria insuficiente para explicar a ampliação da cobertura dos serviços, uma vez que o uso da racionalidade política 15 predominou. Essa hipótese foi levantada a partir dos relatos da maioria dos entrevistados, os quais confirmam que a definição sobre o número e localização das USF a serem implantadas foi estabelecida por meio de reuniões e debates com trabalhadores e usuários nas sedes dos distritos sanitários, com representantes do Conselho Municipal de Saúde e nas plenárias do Orçamento Participativo (OP), realizadas anualmente. Percebe-se, portanto, que a participação social dos grupos, expressa na disputa política no território, influenciou o processo decisório.

De acordo com os relatos da maioria dos entrevistados, a ampliação do PSF no Recife, ocorreu na perspectiva de reorganização do modelo de atenção. Para eles a implantação da central de regulação de consultas especializadas e exames é um dos indícios que corroboram essa intencionalidade da gestão. Com a regulação assistencial, o acesso passou a ocorrer de forma mais ordenada, procurou-se observar as necessidades referidas por cada USF. Portanto, verifica-se que no período estudado, a SMS do Recife buscou promover a articulação entre níveis de atenção, que constitui uma das dimensões da integralidade, 12,13 embora, conforme consenso dos entrevistados, tenha sido insuficiente a oferta de atenção especializada.

Segundo informações do SIA-SUS e SIH-SUS, em 2008, apenas $34,6 \%$ dos procedimentos de média complexidade estavam sob gestão municipal, o que revela uma baixa articulação com a rede de saúde estadual que concentra a maior oferta de tais procedimentos, porém sem a efetiva regulação de acesso, $65,4 \%$ das ações de média e $100 \%$ das de alta complexidade são realizadas no território do Recife.

Em relatos de uma parcela dos entrevistados, verifica-se que a gestão municipal não investiu, de forma substancial, na ampliação de sua governabilidade para proceder à cogestão de leitos hospitalares, tanto eletivos, quanto de urgência e emergência, e no acesso regulado para consultas e exames da população recifense nos serviços sob gestão estadual.

A baixa resolubilidade do PSF, expressa pelo excesso de encaminhamentos à atenção especializada, foi destacada em dois relatos:

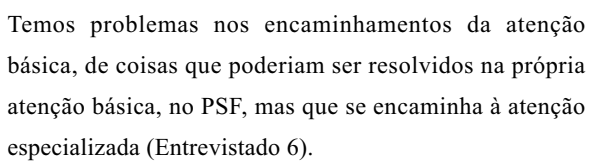

A central municipal de regulação de consultas e exames tem um protocolo de acesso à atenção especializada (da oferta sob gestão municipal), mesmo assim a gente percebe que há um excesso de encaminhamento. Há muitos casos que daria para o PSF resolver (Entrevistado 1)

A resolubilidade do PSF, embora esteja relacionada ao acesso a exames e serviços especializados, 23 também depende de outros de fatores, como, por exemplo, a organização do processo de trabalho e capacitação dos profissionais para identificar e satisfazer as necessidades dos usuários.

Em síntese, constata-se que a política de saúde do Recife, no processo de alocação de recursos assistenciais em áreas mais desassistidas, promoveu a participação social e enfrentou as iniquidades, compreendidas como desigualdades evitáveis, injustas e desnecessárias. ${ }^{5}$ Nesse sentido, restituiu a determinados grupos o acesso a serviços essenciais, incluindo os de saúde. No entanto, problemas de acessibilidade ainda persistem, pois a disponibilidade de recursos é apenas uma das condições básicas do acesso.

\section{A política de saúde do trabalhador no Recife}

Para a maioria dos gestores entrevistados, a expansão dos cuidados de saúde à população trabalhadora foi resultante de discussões entre gestores municipais, movimento sindical e instâncias de controle social. O município incorporou a proposta no Plano Municipal Saúde17 prevendo ações de assistência, vigilância, reabilitação e controle social, numa perspectiva de equidade, integralidade e universalidade do acesso e de inclusão dos trabalhadores da economia informal. 


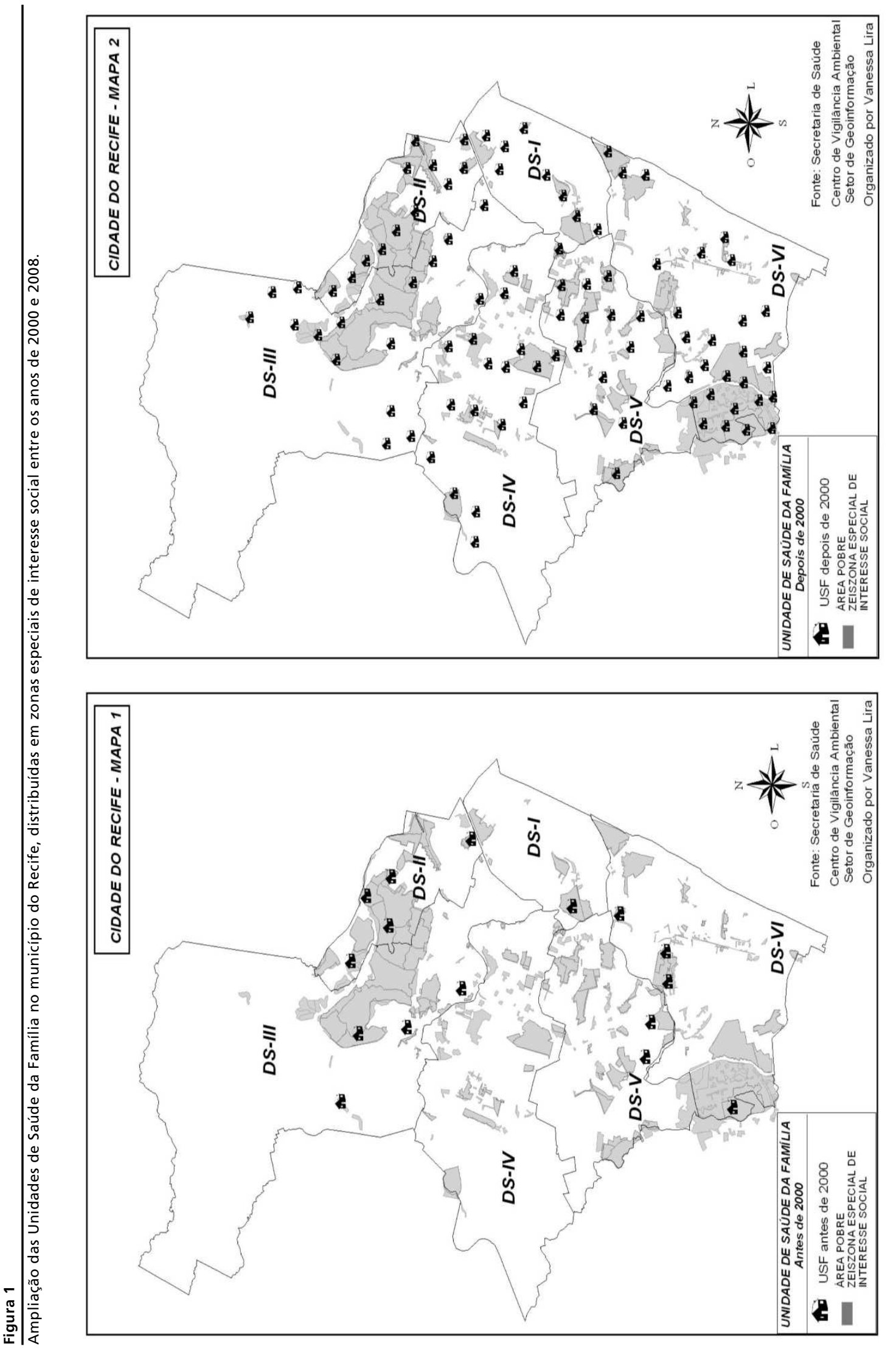


No entanto, durante a implementação da política, como ressaltam todos os entrevistados, o Centro de Referência Regional de Saúde do Trabalhador (CEREST) concentrou-se nas ações assistenciais, sem articulação com outros níveis de atenção, principalmente, com a atenção básica, distanciando-se dos princípios da integralidade da atenção. 12,13

Entre 2002 e 2008, conforme informações do SIA/SUS, o CEREST-Recife realizou apenas 13.228 atendimentos a usuários. A demanda atendida foi majoritariamente proveniente do setor formal, conforme estudo conduzido pela coordenação da política. Dos 679 novos usuários atendidos em 2007, $96,4 \%$ eram do setor formal, $1,8 \%$ do informal e $1,8 \%$ sem informação. 24 Para um parcela dos gestores entrevistados, mesmo havendo articulação do CEREST com representações dos trabalhadores informais (associação dos ambulantes, catadores de material reciclável, dentre outros), as ações específicas não foram consolidadas.

No tocante às ações de vigilância em saúde do trabalhador, pôde-se constatar que as fiscalizações do ambiente de trabalho, decorrentes de demanda espontânea ou programada, não foram plenamente atendidas. Em 2008, a proporção de fiscalizações realizadas em relação ao total de solicitações foi de $53,3 \% .21$ Já a notificação de agravos relacionados ao trabalho, segundo os relatos de parcela dos entrevistados, somente foi efetivamente implantada em meados de 2008. Outrossim, para esses mesmos entrevistados as dificuldades nas atividades de vigilância em saúde do trabalhador têm como causas a qualificação dos profissionais de saúde e atuação política de atores econômicos, como expressa o relato a seguir:

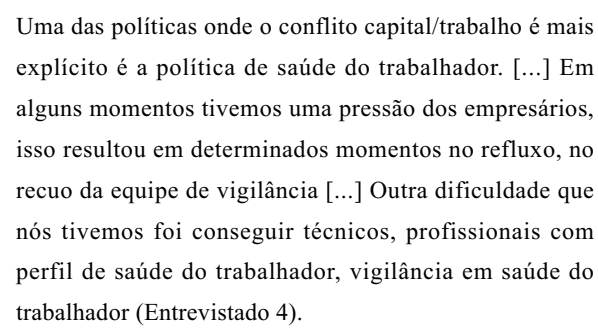

Essa problemática também foi referida por Machado 25 quando analisou as relações entre a temática de saúde do trabalhador e os conflitos capital-trabalho.

Assim, com base nos dados analisados constatase que houve avanços com a implantação da política de saúde do trabalhador no Recife, mas se percebem limitações quanto à equidade e integralidade da atenção.

\section{A inclusão dos portadores de sofrimento psíquico}

A atenção aos portadores de sofrimento psíquico no município até 2001 era desenvolvida de acordo com um modelo asilar, excludente, que privilegiava a internação hospitalar. Na época, havia sete hospitais psiquiátricos sob gestão municipal concentrando $60,2 \%$ do total de leitos do município. Com a mudança de governo em 2001, a Secretaria de Saúde promoveu um processo de desospitalização e implantação de serviços extra-hospitalares, ${ }^{21} \mathrm{em}$ consonância com as propostas do movimento da luta antimanicomial e da sociedade em geral, sobretudo durante a formulação do programa de governo, como relatam alguns dos entrevistados.

Assim, o município implantou uma rede regionalizada de serviços substitutivos com 17 Centros de Atendimentos Psicossocial (CAPS) com cobertura em 2008 de 1,06 por 100 mil habitantes. 26 Incorporou a ideia de que o processo de desconstrução da cultura manicomial passa não apenas pela implantação serviços substitutivos, mas também pela reconstrução das ações no território. Foram implantados mecanismos de desinstitucionalização, por meio da articulação com os profissionais das ESF e com a comunidade, na perspectiva de integralidade da atenção. 12,13 Apesar dos avanços, a integralidade na prática ainda ocorre de forma incipiente e diferenciada entre as ESF, conforme salienta parcela dos gestores entrevistados.

No município, segundo dados do SIH-SUS e SIA-SUS, não houve transferência significativa de recursos financeiros do âmbito hospitalar para o extra-hospitalar, para a atenção aos portadores de sofrimento psíquico, pois, em 2007, os serviços hospitalares consumiram $81 \%$ desses recursos. No nível nacional, no mesmo período, a alocação dos recursos era diferente: os serviços extra-hospitalares absorviam $63,3 \%$ dos recursos contra $36,6 \%$ destinados aos serviços hospitalares. ${ }^{26}$ Além disso, apesar de ter ocorrido, entre 2007 e 2008, uma redução em torno de $28,3 \%$ dos leitos psiquiátricos, o Recife permaneceu como a segunda cidade com maior número de leitos psiquiátricos (1048) do país, segundo informações do Cadastro Nacional de Estabelecimentos de Saúde (CNES), e destacada pelo relato a seguir:

\footnotetext{
$\mathrm{Na}$ verdade não se mexeu justamente na rede que fazia toda a exclusão [...] O grande entrave na saúde mental, que tem uma referência direta na inclusão, é não ter diluído os leitos dos hospitais psiquiátricos. Hoje o Recife continua sendo umas das capitais brasileiras com maior
} 
concentração de leitos em hospitais psiquiátricos (Entrevistado 3)

Para uma parcela dos gestores entrevistados, a saída de atores-chave da gestão, vinculados à luta pela reforma psiquiátrica, contribuiu com a "despolitização" das reformas que vinham sendo implementadas e enfraqueceu a incipiente articulação da gestão com o movimento da luta antimanicomial.

Os achados documentais apontam que o fechamento de dois hospitais psiquiátricos, a implantação de 17 CAPS, a criação de 11 residências terapêuticas (com 88 moradores) e a disponibilização de 50 bolsas ressocialização ${ }^{21}$ foram ações importantes, mas não suficientes para solucionarem o problema da longa permanência de usuários em unidades hospitalares sob gestão municipal. Em 2008, havia 395 pacientes psiquiátricos internados, dos quais $40 \%$ eram procedentes do Recife e $60 \%$ de outros municípios.

A redução das internações de longa permanência, segundo revela a maioria dos gestores entrevistados, depende de um conjunto de ações em âmbito municipal ou locorregional, porque mais de $50 \%$ dos usuários internados em unidades sob gestão municipal são de outros municípios. Nesse sentido, eles ressaltaram a importância de uma atuação articulada entre esferas de governo para implantar ou ampliar redes substitutivas nesses municípios ou regiões do Estado. No entanto, não foi consenso entre os gestores a defesa de propostas relativas à ampliação do número de residências terapêuticas e de CAPS 24 horas ou à disponibilidade de leitos em hospitais gerais.

Com base nos dados analisados, verifica-se que a rede de saúde mental implantada no Recife configurou-se como "alternativa" ao modelo hospitalocêntrico, porém não foi substitutiva, porque a política de saúde mental não consolidou de modo sustentável o processo de desospitalização e de desinstitucionalização dos portadores de sofrimento psíquico.

Saúde da mulher: tecendo as relações entre gênero e raça-etnia

A mulher constituiu um dos grupos sociais que, durante os oito anos de gestão municipal, foi incorporado na agenda de prioridades de governo.17,18 Como destaca a maioria dos entrevistados, um dos protagonistas-chave desse processo foi o movimento de mulheres que manteve uma interlocução sistemática com a gestão no processo de implantação da Política Municipal de Atenção Integral à Saúde da Mulher. $\mathrm{O}$ arco direcional da política fundamentou-se em três eixos: a) assistência humanizada ao pré-natal, parto e aborto e puerpério; b) atenção à mulher vítima de violência doméstica e sexual; c) atenção aos direitos sexuais e reprodutivos, resgatando as questões de gênero. 27

O primeiro eixo ${ }^{27}$ caracterizou-se pelo investimento na reestruturação da atenção com a construção de uma maternidade e reabertura de outras duas, a implantação de doze serviços de referência para pré-natal de alto risco, a instituição do direito da gestante à acompanhante, dentre outras ações. No entanto, mesmo após a ampliação dos leitos obstétricos, as dificuldades de acesso ao parto das mulheres residentes no município permaneceram. Em 2008, segundo dados do SIH/SUS, dos 14.800 partos realizados, apenas $37,0 \%$ ocorrem em maternidades municipais. Dentre esses, $41,7 \%$ foram realizados em unidades sob gestão estadual e 21,3\% em outros municípios. Situação que, para os entrevistados, explica-se pelo fato de os municípios disponibilizarem seus leitos para a Central Estadual de Leitos, mas não participarem de alguma forma da gestão da referida central.

Em termos de oferta de pré-natal, conforme informações da Diretoria de Vigilância à Saúde do Recife, o município manteve, durante o período de estudo, uma cobertura de $88 \%$ (gestantes com quatro consultas e mais), apresentou queda de $46 \%$ da proporção de mulheres que não realização nenhuma consulta pré-natal e elevação do número de gestantes com sete ou mais consulta em torno de $5,6 \%$. Por outro lado, as ações referentes ao pré-natal apresentaram iniquidades raciais, uma vez que no período de 2001-2007. Dentre os 4670 nascidos vivos de mulheres que não realizaram nenhuma consulta prénatal no Recife, $81 \%$ eram filhos de mulheres negras. 28

Tanto na pesquisa documental27,28 quanto nas entrevistas encontraram-se evidências de iniciativas da SMS do Recife em incorporar as questões raciais e étnicas na agenda da política de saúde, com ações de melhoria do registro raça-cor nos sistemas de informações e capacitações no âmbito de várias políticas, incluindo a saúde da mulher. No entanto, como relata uma minoria dos entrevistados, ainda se detectam dificuldades nas condutas dos profissionais de saúde, conforme o relato a seguir:

[...] inicialmente quando se ia fazer o levantamento da área pelos agentes comunitários para a delimitação do território, era muito frequente que eles passassem nas comunidades, nos terreiros e não fizessem identificação daquelas pessoas residentes naquela localidade, naquele 
espaço. Ficavam fora do acesso ao sistema formal de saúde por conta da questão religiosa e o fato de serem religiões originárias da população negra (Entrevistado 2).

Em decorrência do eixo da assistência humanizada ao pré-natal, parto e aborto e puerpério ter práticas consolidadas e hegemônicas, a implantação de ações que demandaram mudanças no processo de trabalho apresentaram, conforme relato de parcela dos entrevistados, algumas dificuldades:

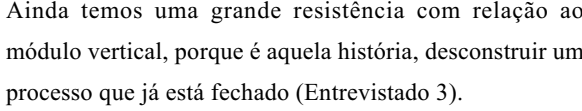

Quanto ao segundo eixo,27 o da atenção à mulher vítima de violência sexual e doméstica, a SMS do Recife implantou um Programa de Atendimento Integral, que incluiu protocolo de atendimento e capacitação dos profissionais da rede básica à atenção especializada. Além disso, estabeleceu um fluxograma de atendimento, construído com a participação de profissionais dos serviços de saúde, da Delegacia da Mulher, do Instituto de Medicina Legal, dos Núcleos de atenção jurídica, e da Casa abrigo.

Esta última constriuiu uma unidade de atendimento implantada pela Secretaria Especial da Mulher com o objetivo de garantir a integridade física, psicossocial e jurídica das mulheres, seus filhos e filhas entre 0 e 12 anos de idade. Além disso, as maternidades municipais foram estruturadas para a realização do aborto legal e para a profilaxia de doenças sexualmente transmissíveis e infecção por HIV (DST/HIV). 27

Com base nesses dados, concluiu-se que a política de saúde da mulher procurou atuar de forma integrada com outras secretarias no enfrentamento da violência contra a mulher, aproximando-se de um dos sentidos da integralidade enquanto integração entre as políticas públicas. ${ }^{12,13}$ No entanto, quanto a assistência ao aborto legal, como ressalta a maioria dos gestores entrevistados, a implantação de protocolos, fluxos e capacitações não tem sido suficiente para romper com as resistências dos profissionais de saúde, conforme se pode observar no relato a seguir:

[...] Na temática do aborto então... na interrupção da gestação, conta-se com pouquíssimas pessoas na hora da intervenção. [...] E se partir do princípio que ela provocou o aborto, aí ela já vai ser a última a ser atendida (Entrevistado 2)

Com base no estudo desenvolvido no Centro de
Assistência Integral à Saúde da Mulher da Universidade Estadual de Campinas, Bedone e Faúndes 29 reforçam a importância de um processo de capacitação sistemático e contínuo das equipes multiprofissionais não enfocando apenas a dimensão do conhecimento técnico, mas a reflexão dos profissionais sobre suas atitudes, conceitos de violência e formas de abordagem no cuidado às vítimas de violência. Para esses autores, a troca de percepções entre os diversos profissionais contribui para a capacitação de cada um e para a eficácia das ações propostas.

Com base nas informações das entrevistas e nos documentos consultados, 21,27 constatou-se ter havido avanços nos direitos sexuais e reprodutivos. A SMS implementou a atenção ao câncer cérvico uterino e de mama, criou uma unidade de referência em mastologia, implantou a anticoncepção de emergência (ACE) na atenção básica e contracepção cirúrgica voluntária nas maternidades municipais, como também implantou a atenção às mulheres no climatério desde atenção básica até a atenção especializada, dentre outras ações.

Por outro lado, o maior desafio do eixo dos direitos sexuais e reprodutivos refere-se à dimensão cultural/ideológica. Um indicador desse processo é a resistência na utilização da contracepção de emergência (CE), encontrada em segmentos religiosos e nos profissionais de saúde, como enfatizado por consenso pelos entrevistados.

Nesse sentido, há evidências de que a política de saúde da mulher no Recife superou a perspectiva reducionista de limitar as necessidades desse grupo populacional às demandas relativas à gravidez e ao parto. Entretanto, por outro lado, verifica-se a existência de dificuldades na implementação das ações para atender a diversidade de necessidades das mulheres. Ainda são pontuais as capacitações para os profissionais de saúde em gênero, raça, etnia e classe social.

\section{Conclusões}

A SMS do Recife reconheceu e procurou satisfazer em sua política de saúde as necessidades de diversos grupos sociais. A alocação de recursos na assistência à saúde em áreas de concentração de pobreza, não restrita apenas à atenção básica; a implantação de uma política de saúde do trabalhador; as mudanças no modelo de atenção aos portadores de sofrimento psíquico e a abrangência das ações de atenção à saúde da mulher para além das demandas relativas à gravidez e ao parto constituem indicadores positivos de transformações que ocorreram na política muni- 
cipal de saúde, caracterizadas pela inclusão de segmentos sociais, portadores de necessidades diversas.

Por outro lado, foram identificadas algumas limitações durante a implementação das políticas destinadas aos grupos sociais. No âmbito da atuação dos profissionais de saúde, as capacitações voltadas ao maior entendimento das questões de gênero, raça, etnia e classe social foram insuficientes. Em relação à organização das ações de saúde, houve predomínio do componente assistencial em detrimento das ações de vigilância à saúde e intersetoriais. $\mathrm{Na}$ relação entre níveis de atenção, observaram-se mecanismos de regulação de acesso a consultas e exames especializados sob gestão municipal, sem ampliação dos espaços de pactuação entre os níveis de gestão pela continuidade do cuidado em outras complexidades assistenciais.

Os resultados revelam que houve uma distância entre os princípios de equidade contidos na formulação da política de saúde e sua operacionalização

\section{Referências}

1. Viana A L, Fausto MCR, Lima LD. Política de saúde e equidade. São Paulo Perspect. 2003; 17: 58-68.

2. Vieira-da-Silva LM, Almeida NF. Equidade em saúde: uma análise crítica de conceitos. Cad Saúde Pública. 2009, 25(Supl. 2): 217-26

3. Cohn A. O SUS e o direito à saúde: universalização e focalização nas políticas de saúde. In: Lima NT, Gerschman S, Edler FC, orgs. Saúde e democracia: história e perspectivas do SUS. Rio de Janeiro: Fiocruz; 2006. p. 385-406.

4. Campos GWS. Reflexões temáticas sobre equidade e saúde: o caso do SUS. Saúde Soc. 2006; 15: 23-33

5. Whitehead M. The concepts and principles of equity in health. Int J Health Serv. 1992; 22: 429-45.

6. Jardaniviski E, Guimarães PCV. O desafio da equidade no setor Saúde. Rev Adm Empres. 1993; 33: 38-51.

7. Sen AK. ¿Por qué la equidad en salud? Rev Panam Salud Publica. 2002; 11: 302-9.

8. Almeida C, Travassos C, Baptista T. A reforma sanitária brasileira: em busca da equidade. Washington, DC Organização Pan Americana de Saúde; 1999. Technical Paper, n. 17

9. Travassos C, Viacava F, Pinheiro R, Brito A. Utilização dos serviços de saúde no Brasil: gênero, características familiares e condição social. Rev Panam Salud Publica. 2002; 11: 365-73.

10. Misoczky MC. Gestão participativa em saúde: potencialidades e desafios para o aprofundamento da democracia Saúde Debate. 2003; 27: 336-47.

11. Escorel S, Moreira MR. Desafios da participação social em saúde na nova agenda da reforma sanitária: democracia deliberativa e efetividade. In: Fleury S, Lobato LVC, orgs. no município. Compreende-se que a troca de gestores, as influências de agências privadas da sociedade civil, a adesão dos profissionais de saúde ao projeto e os interesses conflitantes entre esferas de governo influenciaram no grau dessa implementação.

Assim, constata-se que a SMS do Recife adotou a concepção de equidade vertical, incorporando diversos grupos sociais com necessidades distintas, porém teve dificuldades no atendimento pleno às necessidades de cada um desses grupos, tanto da quantidade das necessidades satisfeitas quanto ao modo como essas necessidades foram atendidas (integralidade da atenção).

Por fim, diante da complexidade da problemática que envolve o tema, ressalta-se a importância de novas investigações que possam envolver não somente o ponto de vista de gestores, mas também dos usuários e/ou grupos sociais e profissionais de saúde.

Participação, democracia e saúde. Rio de Janeiro: Cebes 2009. p. 229-47.

12. Mattos RA. Os sentidos da integralidade: algumas reflexões acerca de valores que merecem ser defendidos. In: Pinheiro R, Mattos RA, orgs. Os sentidos da integralidade na atenção e no cuidado à saúde. Rio de Janeiro: UERJ/ABRASCO; 2001. p. 39-64.

13. Paim JS. Atenção à saúde no Brasil. In: Guimarães R, Angulo-Tuesta A. Saúde no Brasil: contribuições para agenda de prioridades de pesquisa. Brasília, DF: Ministério da Saúde; 2004. p. 15-44

14. Paim JS. Saúde, política e reforma sanitária. Salvador: CEPS/ISC; 2002

15. Rivera FJU. Por um modelo de formulação de políticas de saúde baseado no enforque estratégico da planificação. In: Rivera FJU. Planejamento e programação em saúde: um enfoque estratégico. São Paulo: Cortez; 1992. p. 151-76.

16. Yin RK. Estudo de caso: planejamento e método. 3 ed. Porto Alegre: Bookman; 2005.

17. Recife. Secretaria de Saúde. Plano Municipal de Saúde do Recife: 2002-2005. Recife, 2002.

18. Recife. Secretaria de Saúde. Plano Municipal de Saúde do Recife: 2006-2009. Recife, 2005.

19. Prefeitura da cidade do Recife. Programa das Nações Unidas para o Desenvolvimento. Atlas de desenvolvimento humano de Recife. Recife; 2005.

20. Kvale S. Interviews: an introduction to qualitative research interviewing. London: Sage publications; 1996.

21. Recife. Secretaria de Saúde. Relatório de Gestão 2008. Recife; 2009. 
22. Brasil. Ministério da Saúde. Secretaria de Assistência à Saúde. Departamento de atenção básica. Histórico de cobertura da saúde da família. [Acesso em 12 dez 2009] Disponível em: www.dab.saude.gov.br/abnumeros.php

23. Escorel S, Giovanella L, Magalhães de Mendonça MH, Senna MCM. O Programa de Saúde da Família e a construção de um novo modelo para a atenção básica no Brasil. Rev Panam Salud Publica. 2007; 21: 164-76.

24. Recife. Secretaria de Saúde (Gerência de Atenção à Saúde do Trabalhador). Relatório de Gestão: 2006-2008. Recife, 2008.

25. Machado JMH. Processo de vigilância em saúde do trabalhador. Cad Saúde Pública. 1997; 13: 33-45.
26. Brasil. Ministério da Saúde. Saúde mental em dados 5. Brasília, DF; 2008.

27. Recife. Secretaria de Saúde. Gerencia de atenção à saúde da mulher. Relatório das ações 2001-2008. Recife; 2008.

28. Recife. Secretaria de Saúde (Diretoria de Vigilância à Saúde). Perfil Epidemiológico da criança e do adolescente 2001-2007. Recife; 2008.

29. Bedone A J, Faúndes A. Atendimento integral às mulheres vítimas de violência sexual: Centro de Assistência Integral à Saúde da Mulher, Universidade Estadual de Campinas. Cad Saúde Pública. 2007; 23: 465-9.

Recebido em 23 de março de 2010

Versão final apresentada em 13 de janeiro de 2011

Aprovado em 1 de fevereiro de 2011 